\title{
Non-canonical Hedgehog signaling activation in ovarian borderline tumors and ovarian carcinomas
}

\author{
PETAR OZRETIĆ $^{1 *}$, DIANA TRNSKI $^{1 *}$, VESNA MUSANI $^{1}$, IVANA MAURAC $^{2}$, \\ DRŽISLAV KALAFATIĆ ${ }^{2}$, SLAVKO OREŠKOVIĆ $^{2}$, SONJA LEVANAT $^{1}$ and MAJA SABOL ${ }^{1}$
}

${ }^{1}$ Laboratory for Hereditary Cancer, Division of Molecular Medicine, Ruđer Bošković Institute; ${ }^{2}$ Department of Obstetrics and Gynaecology, University Hospital Centre Zagreb, School of Medicine, University of Zagreb, 10000 Zagreb, Croatia

Received April 27, 2017; Accepted September 19, 2017

DOI: 10.3892/ijo.2017.4156

\begin{abstract}
Hedgehog signaling pathway has been implicated in the pathology of ovarian cancer, and Survivin (BIRC5) has been suggested as a novel target of this pathway. Herein we investigated the role of Hedgehog signaling pathway and Survivin in ovarian carcinoma and borderline tumor samples. We aimed to determine possible ways of pathway modulation on primary ovarian cancer cells and an established cell line. RNA was extracted from fresh tumors and control tissues and gene expression was examined using qRT-PCR. Pathway activity in cell lines was examined after treatment with cyclopamine, SHH protein, GANT-61 or lithium chloride using qRT-PCR, western blot and confocal microscopy. The difference between control tissue, borderline tumors and carcinomas can be seen in GLII and SUFU gene expression, which is significantly higher in borderline tumors compared to carcinomas. SUFU also shows lower expression levels in higher FIGO stages relative to lower stages. BIRC5 is expressed in all tumors and in healthy ovarian tissues compared to our control tissue, healthy fallopian tube samples. Primary cells developed from ovarian carcinoma tissue respond to cyclopamine treatment with a short-term decrease in cell proliferation, downregulation of Hedgehog pathway genes, including BIRC5, and changes in protein dynamics. Stimulation with $\mathrm{SHH}$ protein results in increased cell migration, while GLII transfection or PTCHI silencing demonstrate pathway upregulation. The pathway activity can be modulated by $\mathrm{LiCl}$ at the GSK3 $\beta$-SUFU-GLI level, suggesting at least partial non-canonical activation.
\end{abstract}

Correspondence to: Dr Maja Sabol, Laboratory for Hereditary Cancer, Division of Molecular Medicine, Ruđer Bošković Institute, Bijenička 54, HR-10000 Zagreb, Croatia

E-mail:maja.sabol@irb.hr

*Contributed equally

Key words: ovarian carcinoma, borderline tumors, Hedgehog signaling, cyclopamine, lithium chloride, Survivin
Downregulation of the pathway with GANT-61 has proved to be more effective than cyclopamine. GLI inhibitors may be a superior treatment option in ovarian cancer compared to SMO inhibitors.

\section{Introduction}

Ovarian cancer is the eighth most frequent tumor type in women worldwide, with 225,500 new cases and 140,200 deaths each year. There is almost no difference in mortality between developed and developing countries (1). Epithelial ovarian cancer comprises the majority of malignant ovarian tumors. Borderline epithelial tumors have been classified as a separate category, although their origin is still unclear. Their clinicopathological features are between the benign cystadenomas and malignant cystadenocarcinomas (2). Borderline tumors and low-grade serous carcinomas are associated with KRAS and $B R A F$ mutations. High-grade serous carcinomas are associated with loss of $B R C A 1$ or BRCA2, and with TP53 mutations, but no $B R A F$ and rarely KRAS mutations. This suggests that low-grade and high-grade serous carcinoma arise via different genetic pathways (3). High BIRC5 gene expression (Survivin) is often detected in various tumors, and is correlated with poor outcome. Survivin protein expression correlates with progression of epithelial ovarian carcinomas (4). Epithelial ovarian cancer shows a diverse pattern of genomic rearrangements which lead to altered gene expression and upregulation of cancer pathways, including Hedgehog signaling (5).

Hedgehog signaling pathway is a developmental pathway involved in formation of various tissues and organs. In mammals, the signal transduction is triggered by binding of the ligand Hedgehog $(\mathrm{HH})$ to the transmembrane receptor Patched (PTCH). This leads to internalization of PTCH and exposure of the protein Smoothened (SMO) on the cell surface. SMO triggers a cytoplasmic phosphorylation cascade leading to the release of transcription factor GLI (GLI1-3) from Suppressor of Fused (SUFU) and translocation of GLI to the nucleus, where it triggers transcription of genes involved in cell proliferation. In the absence of the $\mathrm{HH}$ signal, the cascade is inactive and GLI proteins are retained in the cytoplasm and subsequently degraded in the proteasome (6). GLI proteins are regulated by SUFU, protein kinase A (PKA), glycogen synthase kinase-3 $\beta$ (GSK3 $\beta$ ) and casein kinase 1 (CK1). The 
role of GSK3 $\beta$ in the Hedgehog signaling pathway is 2-fold: it phosphorylates GLI proteins, tagging them for degradation and processing into repressor forms, but it can also elicit a positive effect on the signal transduction in ligand-stimulated cells, binding to SUFU and enabling the release of GLI from the complex (7). Genes activated by the Hedgehog pathway are involved in cell cycle regulation, proliferation, adhesion, epithelial-mesenchymal transition, self-renewal and pathway autoregulation. A recent study by Brun et al implicated Survivin in Hedgehog-driven medulloblastoma (8), and a study by Vlčková et al demonstrated a direct link between Survivin expression and Hedgehog signaling, where expression of Survivin was directly regulated by GLI2 and GLI3 proteins (9). Therefore, Survivin is considered a novel Hedgehog pathway target in a large panel of tumor cell lines (9).

The Hedgehog signaling pathway is involved in embryonic development and maturation of Drosophila ovaries. In the adult fly it is involved in division of somatic stem cells which give rise to follicle cells surrounding the gamete. Inactivation of the pathway causes a reduction in follicle production, while hyperactivation causes proliferation of somatic cells between egg chambers (10). In mice, activation of the pathway by Sonic Hedgehog (SHH) protein increases growth and proliferation in cultured granulosa cells, and cyclopamine was shown to prevent this induction. Russell et al claim that many of the Hedgehog pathway genes are expressed in immature and adult murine ovaries, granulosa cells and corpora luteum: $I H H, S H H, D H H, P T C H 1, P T C H 2$ and SMO; while GLII and $H H I P$ are expressed in all ovarian tissues (11). Another more recent study by Huang et al indicates that Hedgehog signaling is inactive in fetal, but active in the adult mouse ovary (12). A paracrine model of signaling was suggested in the ovary: Hedgehog target genes GLII and PTCHI are primarily expressed in theca cells, while the ligand is produced in the granulosa cells $(13,14)$.

According to most authors Hedgehog pathway is activated in ovarian carcinomas, where the protein or gene expression is higher than in healthy tissue or benign tumors (15-18). Other authors report Hedgehog pathway protein expression in a subset of tumors, but claim it is not a frequent event in ovarian cancer (19). The authors mostly focus on the four most frequently examined components of the pathway: SHH, PTCH1, SMO and GLI1. In healthy human ovaries, the Hedgehog pathway genes/proteins are usually not expressed $(15,17)$. Expression of GLI1 and PTCH1 correlates with poor clinical outcome of ovarian carcinoma patients $(16,20)$. Cell culture experiments on ovarian cancer cell lines show that inhibition of Hedgehog signaling leads to inhibition of proliferation, migration and invasion and to apoptosis $(15-17,21,22)$. It has also been demonstrated that inhibition of Hedgehog signaling on the level of GLI1 inhibits the growth of ovarian cancer xenografts $(17,23,24)$, and growth of ovarian cancer spheroids (25).

We and others have shown that loss of heterozygosity (LOH) of the PTCH1 gene is a frequent event in various malignant and benign tumors of the ovary (26-30). Benign tumors, such as dermoids and fibromas, also show methylation of the PTCH1 promoter (31), but this does not seem to be the case for ovarian carcinoma (32).

In this study, we confirm that the Hedgehog signaling pathway is active in ovarian tumors, and that GLII and SUFU are associated with tumor type and FIGO stage. It seems that the activation occurs downstream of the membrane components of the pathway, suggesting non-canonical activation. We also demonstrate the activity in a primary tumor cell line developed from the ovarian tumor sample, which seems to be a superior model for study of the pathway compared to the established lines.

\section{Materials and methods}

Sample collection. Twenty-three samples of ovarian tumors [16 carcinomas (CA), 7 atypical proliferative (borderline) tumors (BDL)] and tissue from 9 healthy ovaries (OV) and 9 healthy fallopian tubes (FT) excised for reasons other than malignant transformation, were collected at the Department of Obstetrics and Gynaecology, University Hospital Centre Zagreb, School of Medicine, University of Zagreb. Of the 16 carcinoma samples, 3 were diagnosed at stage I, 2 at stage II, 7 at stage III and 4 at stage IV according to the International Federation of Gynecology and Obstetrics (FIGO) classification. Of 16 carcinomas, 4 were grade 1 and 12 were grade 3 . By type, 12 were serous adenocarcinoma and 4 were clear cell carcinoma. Of the 7 borderline tumors, 1 was diagnosed at stage III and the remaining 6 were at stage I of FIGO classification. All patients gave their informed consent before the samples were taken, and samples were collected with the approval of the hospital's ethics committee. All tissue samples taken during surgery were immediately placed in a vial containing $1 \mathrm{ml}$ RNALater solution (Invitrogen), kept at $4^{\circ} \mathrm{C}$ overnight, and RNA was extracted using TRIzol reagent (Invitrogen) the following day. RNA concentration was determined spectrophotometrically, and quality was checked on $1 \%$ agarose gel.

Quantitative real-time PCR ( $q R T$-PCR). RNA was reversetranscribed using TaqMan Reverse Transcription Reagents (Applied Biosystems), and qRT-PCR was performed on a CFX96 machine (Bio-Rad) using EVAGreen dye (Bio-Rad). Primers and conditions used were described previously $(33,34)$. Expression levels for each gene were first calculated as $\triangle \mathrm{Ct}$ relative to the housekeeping gene $R P L P O$. Some samples showed no expression of a specific target after 40 cycles of qRT-PCR, but for purposes of statistical tests the $\mathrm{Ct}$ for these samples was set to 40 (35). Expression relative to the healthy fallopian tube tissue $(\Delta \Delta \mathrm{Ct})$ was then calculated using the $2^{-\Delta \Delta C t}$ formula.

Cell culture experiments. Primary culture was established from a high-grade ovarian tumor sample (FIGO IIIC) as previously described (33) and maintained in DMEM+10\%FBS, as was the human ovarian adenocarcinoma cell line SKOV-3. Both cultures were mycoplasma-free. Cells were treated with $7.5 \mu \mathrm{M}$ cyclopamine (Toronto Research Chemicals), $3 \mathrm{ng} / \mu \mathrm{l}$ SHH protein (a kind gift from Professor A. Kenney, USA) for $96 \mathrm{~h}, 2.5$ and $5 \mu \mathrm{M}$ GANT-61 (Selleckchem, S8075) or 5 and $10 \mathrm{mM}$ lithium chloride ( $\mathrm{LiCl}, \mathrm{Kemika}$ ) for $48 \mathrm{~h}$. For MTT assay, primary cells or SKOV-3 cells were plated in 96-well plates, and treated the following day. Cell proliferation was measured after 24,48 and $72 \mathrm{~h}$.

For transfection experiments, SKOV-3 cells were seeded in 6-well plates and transfected the following 

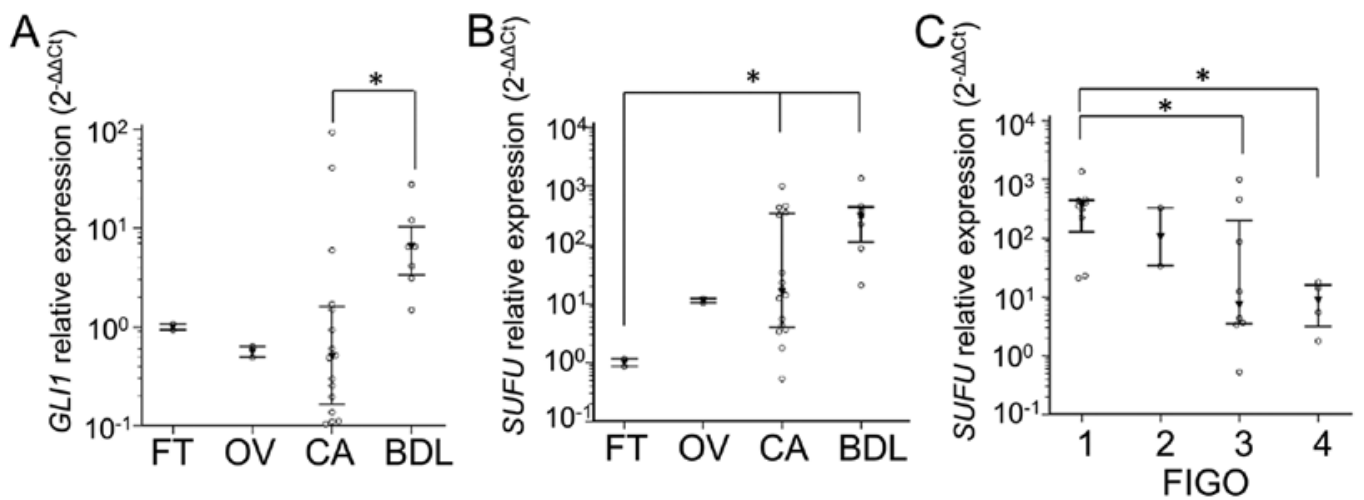

Figure 1. Gene expression in healthy control and ovarian tumor samples represented by log-transformed relative fold change calculated using the $2^{-\Delta \Delta C t}$ formula. (A) Expression of GLII in relation to sample type. (B) Expression of SUFU in relation to sample type. (C) Expression of $S U F U$ in ovarian tumor samples in relation to FIGO stage. FT, healthy fallopian tube; OV, healthy ovary; CA, ovarian carcinoma; BDL, borderline tumor. Clear circles are individual values for each of the samples, black triangle represents the median, and error bars represent the lower to upper quartile (25-75 percentile). ${ }^{*}<<0.05$ pairwise.

day with pcDNA4nISMtGLI1 plasmid (a kind gift from Professor F. Aberger, Austria) using Lipofectamine reagent (Invitrogen). RNA was extracted $24 \mathrm{~h}$ post-transfection and analyzed using qRT-PCR.

For silencing experiments, cells were seeded in 6-well plates and transfected with siPTCH1 (Ambion, Silencer Select s11442) using siPORT reagent (Ambion) in DMEM without serum. After $24 \mathrm{~h}$, the transfection was repeated, and $24 \mathrm{~h}$ later medium was changed to DMEM $+10 \% \mathrm{FBS}$. For negative control, cells were transfected with negative control siRNA (Ambion, Silencer Select Negative Control \#1 siRNA) in the same way. Cells were left to recover for additional $24 \mathrm{~h}$ and then RNA was extracted.

For migration analysis, cells were starved for $24 \mathrm{~h}$ in media without serum, and plated in Transwell migration chambers with or without cyclopamine $(7.5 \mu \mathrm{M})$ or $\mathrm{SHH}$ protein (3 $\mathrm{ng} / \mu \mathrm{l})$. Serum-containing media (10\% FBS) was used as chemoattractant in the lower chamber. Cells were fixed and stained with crystal violet $24 \mathrm{~h}$ (for the primary cells) or $4 \mathrm{~h}$ (for SKOV-3) after plating, since SKOV-3 were too migratory after $24 \mathrm{~h}$ and all plated cells migrated across the membrane. Minimum of 5 optical fields at x400 magnification were counted for each experiment.

Western blotting. Total proteins were extracted using standard methods and $50 \mu \mathrm{g}$ was loaded on polyacrylamide gel. Primary antibodies were rabbit polyclonal anti-GLI3 (ProteinTech, 19949-1-AP, 1:600), rabbit polyclonal anti-GLI2 (Aviva Systems Biology, ARP31885_T100, 1:1000), rabbit polyclonal anti-PTCH1 (ProteinTech, 17520-1-AP, 1:600), mouse monoclonal anti-GSK3 $\beta$ (Cell Signaling Technology, \#9832, 1:1000), rabbit monoclonal anti-phospho-Ser9 GSK3 $\beta$ (Cell Signaling Technology, \#5558, 1:1000) and mouse monoclonal anti- $\beta$ actin (ProteinTech, 60008-1-Ig, 1:2000). Secondary antibodies were HRP-conjugated anti-rabbit (Santa Cruz Biotechnology, sc-2370, 1:2000) and anti-mouse (GE Healthcare, NA931V, 1:2000), and the signal was detected using SuperSignal West Pico and Femto (Thermo Fisher Scientific).

Immunofluorescent staining. Cells were grown on cover slides in DMEM complemented with $10 \%$ FBS and treated with $7.5 \mu \mathrm{M}$ cyclopamine for $24 \mathrm{~h}$. Cells were paraformal- dehyde-fixed, permeabilized with methanol and heated in epitope-retrieval solution at $85^{\circ} \mathrm{C}$ for $10 \mathrm{~min}$. The primary antibodies diluted 1:100 were: rabbit polyclonal anti-GLI1 (Santa Cruz Biotechnology, sc-20687), goat polyclonal antiSUFU (Santa Cruz Biotechnology, sc-10933), rabbit polyclonal anti-HH (Santa Cruz Biotechnology, sc-9024) and goat polyclonal anti-PTCH1 (Santa Cruz Biotechnology, sc-6147). Secondary antibodies, at 1:100 dilution, were mouse antigoat-FITC (Santa Cruz Biotechnology, sc-2356) and donkey anti-rabbit-TR (Santa Cruz Biotechnology, sc-2784). Nuclei were stained with DAPI. Negative control slides were treated in the same way omitting the primary antibody. Co-localization coefficient was determined using the ImageJ software v.1.45e with the Co-localization test plugin.

Statistical analysis. D'Agostino-Pearson test was used for testing the normality of the data distribution. Since data did not show a Gaussian distribution, non-parametric tests were used for statistical analyses. Kruskal-Wallis test with a posthoc test for pairwise comparison of subgroups according to Conover were used to determine the association between gene expression (Ct values) and tumor type (ovarian carcinoma or borderline), FIGO stages (I-IV) or grade. Correlations between gene expression levels were assessed by Spearman's rank correlation coefficient $\varrho$ and ranked according to Colton (36). Mann-Whitney test was used for comparing co-localization coefficients, while Kruskal-Wallis test was used to compare treated and non-treated values for the migration assay. Two-tailed P-values $<0.05$ were considered to indicate statistically significant differences. Statistical analyses were performed using MedCalc for Windows v.15.8 (MedCalc Software bvba).

\section{Results}

Gene expression of Hedgehog pathway genes (PTCH1, SMO, GLI1, GLI2, GLI3, SHH, SUFU) and tumor survival marker Survivin (BIRC5), was examined in tumor samples (CA or BDL), healthy ovary (OV) and healthy fallopian tube (FT). Hedgehog pathway genes were found expressed in the majority of ovarian tumor samples: PTCHI (23/23, 100\%), SMO (23/23, 100\%), GLII (17/23, 73.9\%), GLI2 (17/23, 73.9\%), GLI3 (23/23, 
Table I. Correlation of gene expression and FIGO stage in ovarian tumors.

\begin{tabular}{|c|c|c|c|c|c|c|c|c|c|}
\hline GLI2 & GLI3 & PTCHI & SHH & $S M O$ & $S U F U$ & BIRC5 & $F I G O$ & & $\begin{array}{l}\text { Correlation } \\
\text { coefficient }^{\mathrm{a}}\end{array}$ \\
\hline 0.30 & 0.26 & 0.45 & 0.61 & 0.71 & 0.39 & 0.06 & -0.42 & GLII & $\varrho$ \\
\hline \multirow[t]{15}{*}{0.165} & 0.224 & 0.034 & 0.002 & 0.0002 & 0.069 & 0.795 & 0.047 & & P-value \\
\hline & 0.70 & 0.67 & 0.04 & 0.38 & 0.61 & 0.43 & -0.37 & GLI2 & $\varrho$ \\
\hline & 0.0002 & 0.0005 & 0.851 & 0.073 & 0.002 & 0.040 & 0.081 & & P-value \\
\hline & & 0.53 & 0.04 & 0.37 & 0.42 & 0.29 & -0.20 & GLI3 & $\varrho$ \\
\hline & & 0.010 & 0.869 & 0.084 & 0.044 & 0.187 & 0.364 & & P-value \\
\hline & & & -0.04 & 0.61 & 0.85 & 0.53 & -0.54 & PTCHI & $\varrho$ \\
\hline & & & 0.872 & 0.002 & $<0.0001$ & 0.010 & 0.008 & & P-value \\
\hline & & & & 0.43 & 0.11 & -0.39 & -0.13 & SHH & $\varrho$ \\
\hline & & & & 0.039 & 0.612 & 0.066 & 0.557 & & P-value \\
\hline & & & & & 0.62 & 0.22 & -0.33 & $S M O$ & $\varrho$ \\
\hline & & & & & 0.002 & 0.321 & 0.127 & & P-value \\
\hline & & & & & & 0.51 & -0.60 & SUFU & $\varrho$ \\
\hline & & & & & & 0.012 & 0.002 & & P-value \\
\hline & & & & & & & -0.22 & BIRC5 & $\varrho$ \\
\hline & & & & & & & 0.314 & & P-value \\
\hline
\end{tabular}

${ }^{\text {aS }}$ pearman rank correlation coefficient $\varrho$. Statistically significant $\mathrm{P}$-values are in bold.

$100 \%), \operatorname{SHH}(21 / 23,91.3 \%)$ and SUFU (22/23, 95.7\%). GLII was not detected in OV or FT, while all the other genes were detected in both control tissues. BIRC5 expression was used as a tool for deciding on appropriate control tissue, since it is differentially expressed in healthy tissue compared to tumor tissue (4) and its downregulation has potential application in ovarian tumor therapy (37). BIRC5 was upregulated in both tumor types (22/23 samples, $95.7 \%)$, as well as in healthy ovaries, compared to the fallopian tube tissue where it was not detected. Therefore we decided that fallopian tube represents a better control tissue for ovarian carcinomas, which also corresponds to recent literature data (38). GLII and SUFU were the only differentially expressed genes. Expression of GLII was significantly higher in BDL compared to CA $(\mathrm{P}=0.042)$ (Fig. 1A). The same was observed for $\operatorname{SUFU}(\mathrm{P}=0.025)$ (Fig. 1B). When ovarian tumors were classified according to FIGO, we showed that higher FIGO stages had lower expression of $S U F U(\mathrm{P}=0.044)$ (Fig. 1C). Other tested Hedgehog pathway genes were not associated with either tumor type or FIGO, but similarly expressed between the categories. There was no difference in gene expression levels in carcinoma samples regarding the grade (data not shown).

Significant correlation was detected between the tested genes in the dataset of ovarian carcinoma samples. Weak or moderate correlation was detected between many components of the Hedgehog signaling, with excellent correlation between PTCH1 and SUFU ( $\mathrm{Q}=0.85, \mathrm{P}<0.0001)$. Hedgehog signaling genes were also positively correlated with $B I R C 5$ expression (GLI2, PTCH1 and SUFU). The correlation table also confirms the negative correlation between FIGO status and SUFU but also GLII and PTCHI expression (for details see Table I).

To determine if the pathway is fully functional in ovarian tumors, we established a primary culture from one of the tumor samples (FIGO IIIC). Cyclopamine shows an inhibitory effect on primary cell growth $24 \mathrm{~h}$ after treatment, but cells quickly recover and achieve the proliferation equal to nontreated cells after 48-72 h (Fig. 2A). The effect on primary culture is stronger than on the ovarian tumor cell line SKOV-3, although the pattern is similar (Fig. 2B). SHH protein shows no additional proliferative effect on either primary cells or the SKOV-3 cell line (Fig. 2A and B). On gene expression level, cyclopamine treatment of the primary culture induces significant downregulation of Hedgehog pathway genes PTCH1, GLII and GLI2, while GLI3 remains mostly unchanged (Fig. 2C). The gene expression level for the tested genes remains unchanged in cylopamine-treated SKOV-3 cell line (Fig. 2D). SHH treatment of the primary culture shows no change in gene expression of most pathway genes, except an upregulation of GLI2 expression (Fig. 2C). The SKOV-3 cell line is also mostly unresponsive to $\mathrm{SHH}$ stimulation, and even shows downregulation of GLI2 and GLI3 genes (Fig. 2D).

To establish the effect of GLI1 upregulation, cells were transiently transfected with GLII. The primary culture was difficult to transfect, and only a 20-fold increase in GLII expression was achieved. This proved to be too weak to demonstrate the effect of pathway activation, but a slight upregulation was detected (Fig. 2E). Therefore we tested the effect of GLII transfection on SKOV-3 cell line, which was easier to transfect. Apart from the expected upregulation of GLII, other Hedgehog signaling 

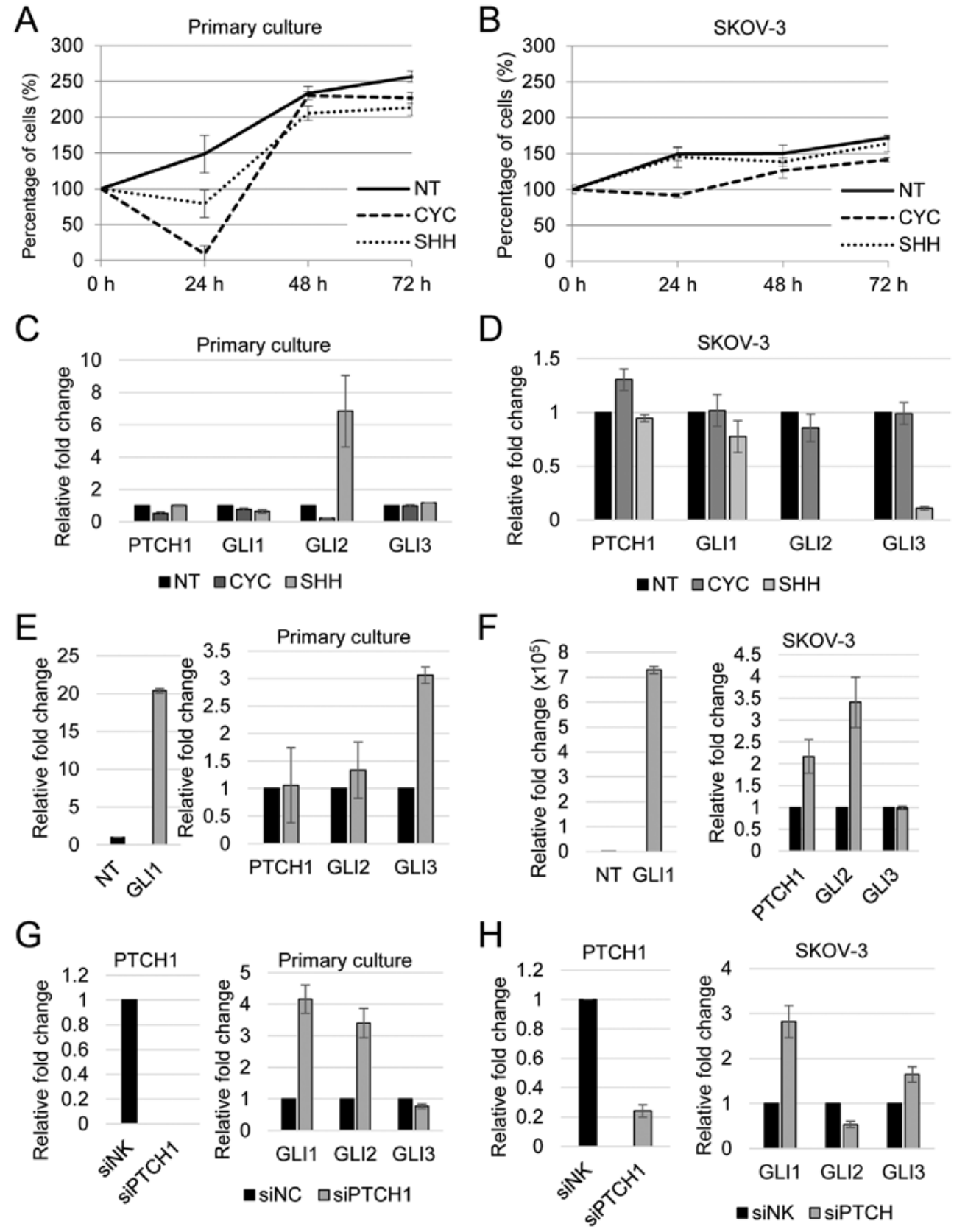

Figure 2. Effect of canonical Hedgehog signaling pathway modulation on ovarian cancer cells. MTT proliferation assay in ovarian cancer primary culture (A) and SKOV-3 cell line (B) shows a slight inhibitory effect of cyclopamine in the first $24 \mathrm{~h}$, after which cells recover to normal levels. Shh treatment shows no proliferative effect on either line. The full line represents the proliferation of non-treated cells (NT), dashed line represents proliferation after cyclopamine treatment (CYC) and dotted line is after SHH protein treatment (SHH). The effect of cyclopamine and Shh treatment on primary cells $(\mathrm{C})$, and the carcinoma cell line SKOV-3 (D). Transfection with GLII upregulates signaling in both the primary culture (E) and the SKOV-3 cell line (F). Gene expression after PTCHI silencing (siPTCH1) in the primary culture and SKOV-3 cell line compared to the scrambled negative control silencing siRNA (siNK). For primary culture, PTCH1 mRNA levels were undetectable after 40 cycles of qRT-PCR, while in SKOV-3 cell line the expression was reduced to 24\%. Both primary (G) and SKOV-3 (H) cell lines show upregulation of GLI upon PTCH1 silencing. Expression in control cells (siNK or NT) was considered 1 for each gene, and relative fold change after transfection was calculated using the $2^{-\Delta \Delta C \mathrm{C}}$ formula.

pathway genes $P T C H 1$ and GLI2, are also upregulated after transfection with GLII. GLI3 levels remain unaltered, showing upregulation of Hedgehog pathway activity (Fig. 2F).

PTCH protein is the negative regulator of Hedgehog signaling. Therefore, PTCH1 silencing should increase Hedgehog functional pathway activity in cells. In the primary cell line, upon PTCH1 silencing GLII and GLI2 are upregulated and GLI3 downregulated (Fig. 2G). For the SKOV-3 cell line, GLII is also upregulated upon PTCH1 silencing, but GLI2 is downregulated while GLI3 is upregulated (Fig. $2 \mathrm{H}$ ).
Immunofluorescent staining of the primary cells shows nuclear localization of GLI1 in non-treated conditions, while SUFU is located mostly at the nuclear periphery and the cytoplasm. After treatment with cyclopamine GLI1 shows a tendency to move to the nuclear periphery and cytoplasm and co-localizes with SUFU (Fig. 3A), with significant increase of the co-localization coefficient $(\mathrm{P}=0.0495)$ (Fig. 3C). In nontreated conditions $\mathrm{SHH}$ shows cytoplasmic localization, while PTCH1 localizes to the cytoplasm with slightly more intense staining in the region of SHH, suggesting their interaction. 

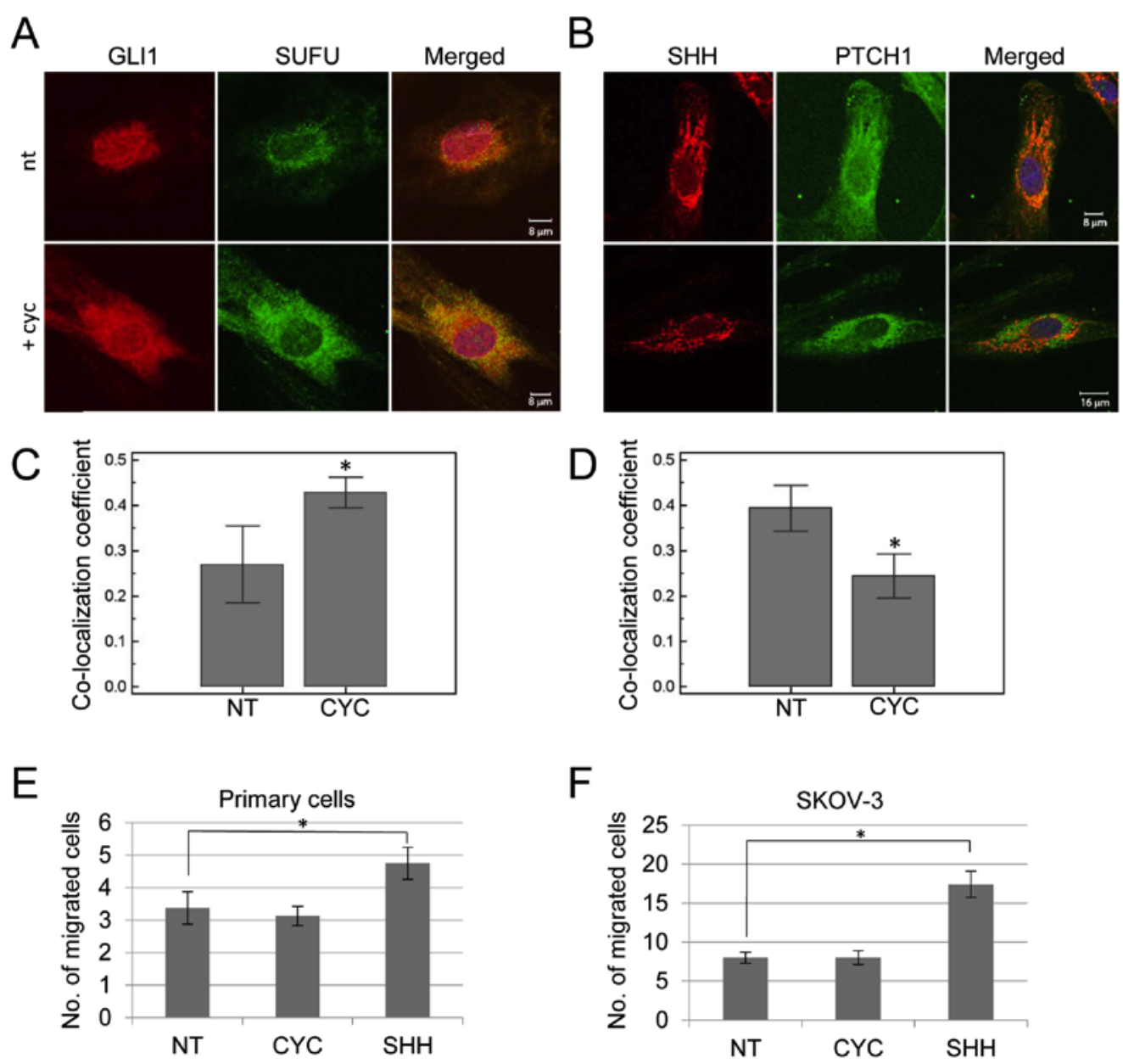

Figure 3. Effect of cyclopamine on protein localization. In primary cells, GLI1 is located in the nucleus in non-treated conditions, but after cyclopamine treatment the staining of GLI1 is predominantly in the cytoplasm where it co-localizes with SUFU (A, C). SHH and PTCH1 co-localize in non-treated conditions, but after cyclopamine treatment this co-localization is reduced (B, D). SHH protein treatment results in an increase in cell migration in both primary cells (E) and SKOV-3 cell line (F). ${ }^{*} \mathrm{P}<0.05$ compared with the control group.

After cyclopamine treatment PTCH1 is located in different parts of the cytoplasm and ceases to co-localize with $\mathrm{SHH}$ ( $\mathrm{P}=0.021)$ (Fig. 3B and D).

Although SHH protein treatment shows no effect on the level of gene expression of Hedgehog pathway genes, its effect is noticeable in migration assays. SHH protein treatment results in significant increase in cell migration in both the primary cells and in the SKOV-3 cell line $(\mathrm{P}=0.045$ and $\mathrm{P}=0.009$, respectively), while cyclopamine shows no effect on cell migration (Fig. 3E and F).

Since cyclopamine treatment affected both the primary and SKOV-3 cell lines only in the first $24 \mathrm{~h}$ of treatment, after which their proliferation rate returned to the level of non-treated cells we decided to test for potential non-canonical activation. We tested the responsiveness of cells to pathway modulation downstream of SMO. Lithium chloride is an inhibitor of GSK3 $\beta$ (39), a protein kinase involved in GLI protein processing into activator or repressor forms, while GANT-61 is a specific GLI inhibitor. Both $\mathrm{LiCl}$ and GANT-61 decrease the proliferation rates of the primary and SKOV-3 cell lines after $72 \mathrm{~h}$ (Fig. 4A and $\mathrm{B}$ ). On the protein level, it can be noted that $\mathrm{LiCl}$ increases the Ser9 phosphorylated fraction of GSK $3 \beta$ in both cell lines as we expected, while there is almost no change in total GSK3 $\beta$ levels in either cell line (Fig. 4C). Our laboratory has previously shown that dysregulated GSK3 $\beta$ can cause upregulation of the Hedgehog pathway (40) and increasing the fraction of Ser9 phosphorylated GSK $3 \beta$ promotes GLI3 processing into the transcriptional repressor form and subsequent downregulation of the pathway. After treatment with $\mathrm{LiCl}$ a decrease in the levels of GLI3 activator form (GLI3A) can be noted in both cell lines. The GLI3 repressor form (GLI3R) was undetectable under these conditions in the SKOV-3 cell line, but in the primary cell line an increase in GLI3R levels can be observed after treatment with $10 \mathrm{mM} \mathrm{LiCl}$ (Fig. 4C). Moreover, levels of PTCH1, a marker of Hedgehog pathway activity, are decreased in both cell lines after $\mathrm{LiCl}$ treatment, indicating signaling downregulation (Fig. 4C). GLI inhibition with GANT-61 causes a decrease in GLI2 protein levels in both the primary and SKOV-3 cell line, as well as a decrease in the levels of PTCH1 protein indicating pathway downregulation. A decrease in GLI3A levels is detected in both cell lines, and a decrease in GLI3R levels in the primary cell line (Fig. 4D). GLI1 is weakly expressed in both cell lines and was undetectable on western blotting.

\section{Discussion}

Hedgehog pathway genes are expressed in the majority of ovarian tumor samples, with GLII and SUFU differing between 


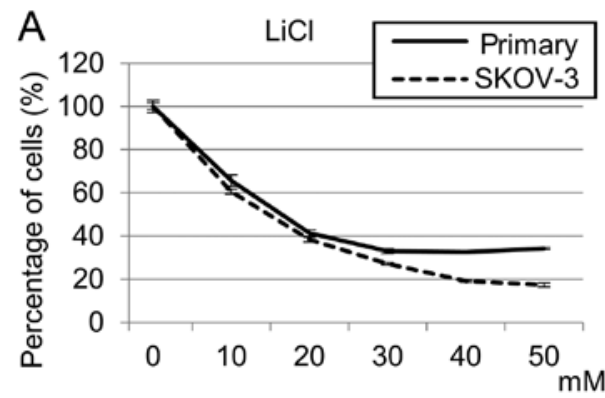

B
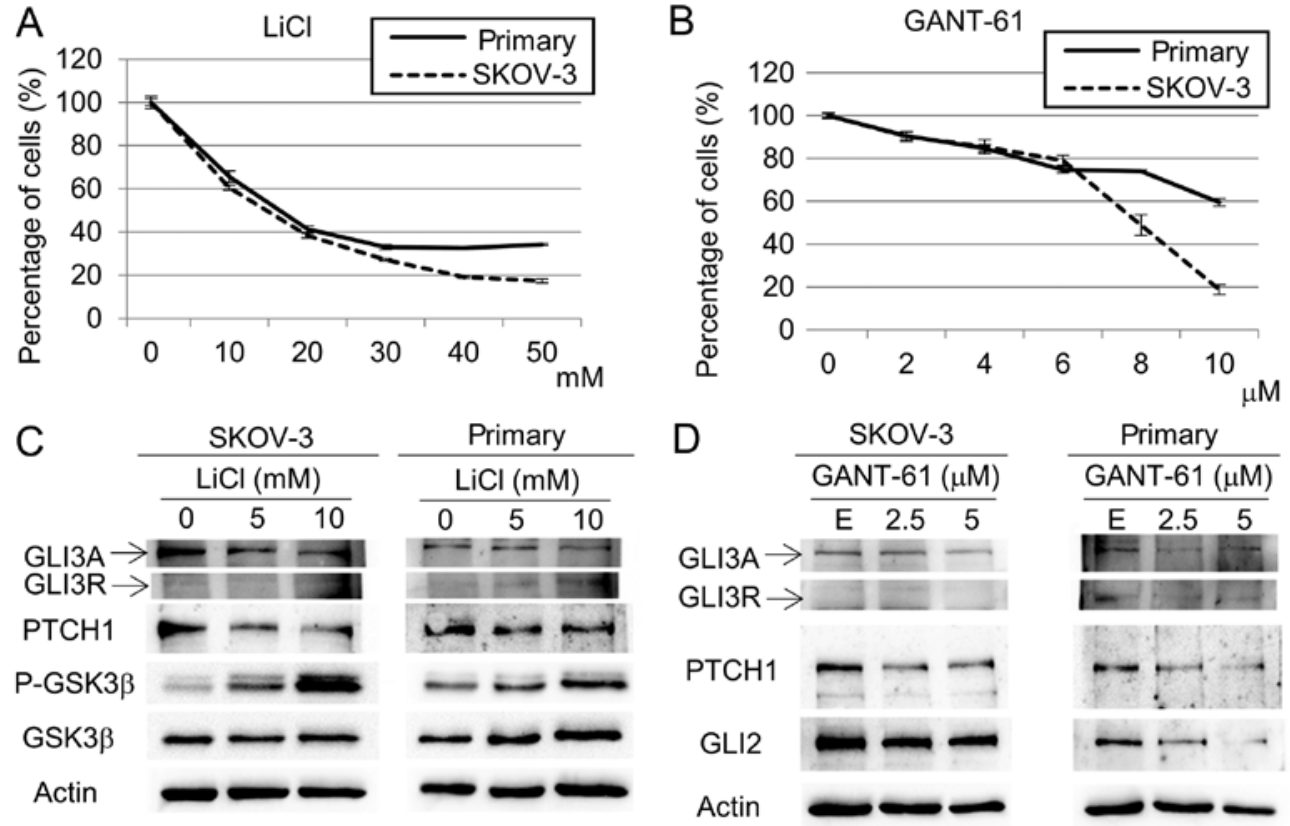

Figure 4. Effect of lithium chloride and GANT-61 treatment on ovarian cancer cells. Both the primary and the SKOV-3 cell lines show dose-dependent mortality upon treatment with $\mathrm{LiCl}$ (A) or GANT-61 (B). LiCl treatment increases the fraction of phosphorylated GSK3 $\beta$ protein, while total GSK3 $\beta$ levels remain unaltered. The same treatment downregulates PTCH1 and GLI3 protein levels (C). GANT-61 treatments downregulates GLI2 and consequently PTCH1 protein levels in both lines (D). E, ethanol.

tumor types, and SUFU between FIGO stages. Majority of studies examining Hedgehog signaling used healthy ovaries as control tissue, which makes sense when examining the tumors immunohistochemically. However, ovarian carcinoma can arise from either the fallopian tube or the ovary itself, with each tissue giving rise to different tumor type (41). For this research we used both types of controls: healthy ovary samples and healthy fallopian tube samples. For our purposes FT controls seem a better control than the OV samples, although the differences between the two control groups were mostly not significant. The only difference between FT and OV groups was observed for BIRC5 expression, where FT has no expression of the gene, while OV expresses it. Since Survivin is a protein associated with normal function of mitotic spindle, and the ovarian tissue undergoes periodic cycles of cell division, some expression of the protein is to be expected in OV.

There is not much data on Hedgehog pathway expression in the healthy human ovary. The groups that used OV as control tissue for immunohistochemical staining report no or weak signal of Hedgehog pathway proteins $(15,17,20)$. Studies on mouse ovaries show reactivation of the pathway in the adult ovary (12). Since tissues in the adult ovary go through periodical phases of proliferation, it is not surprising to detect Hedgehog pathway activity. Still, we did not detect the major effector of the pathway, GLII, in the OV or FT. Given that expression of $P T C H 1$ and $S M O$ were detected, it is possible that in these tissues the Hedgehog signaling is poised for reactivation, and requires a specific stimulus to become active. The key proteins that seem to be involved in pathway upregulation in clinical samples are GLI1 and SUFU protein, suggesting non-canonical activation of the pathway in ovarian tumors. The expression of pathway genes GLI2, PTCH1 and $S U F U$ is correlated with BIRC5 expression in these samples. This, taken together with the recent work by Vlčkova et al (9), suggests that Hedgehog signaling regulates BIRC5 expression in ovarian tumors as well. Our group has previously demonstrated a very similar correlation of Hedgehog signaling with $B I R C 5$ expression in oropharyngeal squamous cell carcinoma (PTCH1 positively and $\mathrm{SHH}$ negatively correlated with $\mathrm{BIRC5}$ expression) (34).

Vlčkova et al also demonstrated that the major regulator of BIRC5 expression in their luciferase model system is GLI2 protein, followed by GLI3 (9). In our samples we detect the expression of all three GLI genes, but the only one that seems to be associated with tumor progression is GLII. However, it must be taken into account that regulation of activity of GLI proteins occurs on post-translational level, so gene expression may not be the best choice for monitoring these effects. Protein expression, on the other hand, is limited by quality of antibodies currently on the market, and their inability to distinguish between activator and repressor forms on tissue sections.

Experiments on primary cells developed from ovarian carcinoma tissue demonstrate that the Hedgehog signaling pathway can be temporarily downregulated using cyclopamine, and cell proliferation is delayed but not completely inhibited. This is further supported by the retention of GLI1 and SUFU in the cytoplasm and reduced PTCH1-SHH co-localization following cyclopamine treatment. SKOV-3 cell line also shows a weak effect of cyclopamine on cell proliferation (a slight delay), but no effect on gene expression level. Exogenous SHH stimulation shows no direct effect on gene expression of Hedgehog pathway genes, but it positively affects the migratory potential of the cells. It is possible that the SHH ligand binds to an alternative target and stimulates cell migration, as we have shown recently on breast cancer (42), and this effect should be investigated further. Endogenous stimulation of the pathway by GLI1 results in upregulation of PTCHI and GLI2/3. Silencing of PTCH1, a negative regulator of the pathway, also upregulates signaling, 
especially $G L I$ transcription factors. These findings suggest that the Hedgehog signaling in ovarian tumors is activated in a noncanonical manner, at least partially bypassing the ligand-receptor signal and instead activating the SUFU-GLI1 axis directly.

Inhibition of SMO by cyclopamine has a short-term effect which blocks the canonical pathway, but the activity is retained through the non-canonical signaling. Inhibitors targeting GLI proteins or their regulators may be more relevant in this context. One potential candidate compound is $\mathrm{LiCl}$, which acts on GSK3 $\beta$, one of the regulators of GLI processing. It has been demonstrated previously that GSK $3 \beta$ usually acts as a tumor suppressor gene, but if it is overexpressed and dysregulated it can have tumorigenic properties, for example in colon cancer $(43,44)$. This effect was also observed in ovarian cancer cells $(45,46)$. Herein, we observed that treatment with $\mathrm{LiCl}$ increases the Ser9 phosphorylated fraction of GSK3 $\beta$ in both cell lines. As a consequence the levels of GLI3 protein decreased in both cell lines. However, the effect of increased processing of GLI3 into repressor form was only observed in the primary cell line. Nevertheless, both cell lines showed downregulated Hedgehog signaling, after $\mathrm{LiCl}$ treatment, indicated by decreased PTCH1 protein levels. The effect of GLI inhibition with GANT-61 was very similar to the effect of $\mathrm{LiCl}$. GANT-61 inhibits GLI1 and GLI2 proteins but according to literature does not affect GLI3 $(47,48)$. The proliferation rate of both cell lines decreased after treatment with GANT-61, and the Hedgehog signaling pathway was downregulated. GANT-61 significantly decreased GLI2 protein levels in both cell lines, which in turn caused a decrease in PTCH1 protein levels. These results indicate that the activity of Hedgehog signaling pathway can be modulated through GSK $3 \beta$ inhibition in ovarian cancer cells, as well as through direct GLI inhibition.

Downstream effectors play a role in regulation of GLI proteins, and may be the key to successful Hedgehog pathway modulation. For example, it has recently been shown that diindolylmethane inhibits GLI1 and leads to anoikis of ovarian cancer cells (24), and GLI proteins are becoming preferred therapy targets since they can inhibit both canonical and noncanonical Hedgehog signaling (49). All these results signify that the signaling pathway is fully functional in ovarian carcinoma primary cells and could be targeted by compounds modifying Hedgehog signaling. Finally, primary cell cultures seem to be a superior model compared to the cell line SKOV-3. It has been shown that ovarian cancer cell lines show great genomic instability, which definitely affects their reliability in cell culture experiments and explains the discrepancy in results obtained by different research groups (50). The primary culture more accurately reflects conditions in vivo, but has a limited lifespan.

\section{Acknowledgements}

This work was funded by the Croatian Ministry of Science, Education and Sports, grant no. 098-0982464-2461, the City of Zagreb, URBROJ: 251-03-02-15-2, and the Croatian Society for Gynecological Urology. The authors wish to thank all the patients who participated in this study. We also thank Professor Fritz Aberger for the GLI1 expression vector used in the study, Professor Anna M. Kenney for the recombinant SHH protein, and Lucija Horvat, BSc for help with confocal microscopy.

\section{References}

1. American Cancer Society: Global Cancer Facts \& Figures. 2nd Edition. American Cancer Society, Atlanta, GA, 2011. https://www.cancer.org/content/dam/cancer-org/research/ cancer-facts-and-statistics/global-cancer-facts-and-figures/ global-cancer-facts-and-figures-2nd-edition.pdf.

2. Hart WR: Borderline epithelial tumors of the ovary. Mod Pathol 18 (Suppl 2): S33-S50, 2005.

3. Bell DA: Origins and molecular pathology of ovarian cancer. Mod Pathol 18 (Suppl 2): S19-S32, 2005.

4. Takai N, Miyazaki T, Nishida M, Nasu K and Miyakawa I: Expression of survivin is associated with malignant potential in epithelial ovarian carcinoma. Int J Mol Med 10: 211-216, 2002.

5. Hoogstraat M, de Pagter MS, Cirkel GA, van Roosmalen MJ, Harkins TT, Duran K, Kreeftmeijer J, Renkens I, Witteveen PO, Lee CC, et al: Genomic and transcriptomic plasticity in treatment-naive ovarian cancer. Genome Res 24: 200-211, 2014.

6. Huntzicker EG, Estay IS, Zhen H, Lokteva LA, Jackson PK and Oro AE: Dual degradation signals control Gli protein stability and tumor formation. Genes Dev 20: 276-281, 2006.

7. Takenaka K, Kise Y and Miki H: GSK3beta positively regulates Hedgehog signaling through Sufu in mammalian cells. Biochem Biophys Res Commun 353: 501-508, 2007.

8. Brun SN, Markant SL, Esparza LA, Garcia G, Terry D, Huang JM, Pavlyukov MS, Li XN, Grant GA, Crawford JR, et al: Survivin as a therapeutic target in Sonic hedgehog-driven medulloblastoma. Oncogene 34: 3770-3779, 2015.

9. Vlčková K, Ondrušová L, Vachtenheim J, Réda J, Dundr P, Zadinová M, Žáková P and Poučková P: Survivin, a novel target of the Hedgehog/GLI signaling pathway in human tumor cells. Cell Death Dis 7: e2048, 2016.

10. Zhang Y and Kalderon D: Hedgehog acts as a somatic stem cell factor in the Drosophila ovary. Nature 410: 599-604, 2001.

11. Russell MC, Cowan RG, Harman RM, Walker AL and Quirk SM: The hedgehog signaling pathway in the mouse ovary. Biol Reprod 77: 226-236, 2007.

12. Huang C-CJ and Yao HH-C: Diverse functions of Hedgehog signaling in formation and physiology of steroidogenic organs. Mol Reprod Dev 77: 489-496, 2010.

13. Wijgerde M, Ooms M, Hoogerbrugge JW and Grootegoed JA: Hedgehog signaling in mouse ovary: Indian hedgehog and desert hedgehog from granulosa cells induce target gene expression in developing theca cells. Endocrinology 146: 3558-3566, 2005.

14. Spicer LJ, Sudo S, Aad PY, Wang LS, Chun SY, Ben-Shlomo I, Klein C and Hsueh AJ: The hedgehog-patched signaling pathway and function in the mammalian ovary: A novel role for hedgehog proteins in stimulating proliferation and steroidogenesis of theca cells. Reproduction 138: 329-339, 2009.

15. Chen X, Horiuchi A, Kikuchi N, Osada R, Yoshida J, Shiozawa T and Konishi I: Hedgehog signal pathway is activated in ovarian carcinomas, correlating with cell proliferation: It's inhibition leads to growth suppression and apoptosis. Cancer Sci 98: 68-76, 2007.

16. Liao X, Siu MKY, Au CWH, Wong ES, Chan HY, Ip PP, Ngan HY and Cheung AN: Aberrant activation of hedgehog signaling pathway in ovarian cancers: Effect on prognosis, cell invasion and differentiation. Carcinogenesis 30: 131-140, 2009.

17. Bhattacharya R, Kwon J, Ali B, Wang E, Patra S, Shridhar V and Mukherjee P: Role of hedgehog signaling in ovarian cancer. Clin Cancer Res 14: 7659-7666, 2008.

18. Schmid S, Bieber M, Zhang F, Zhang M, He B, Jablons D and Teng NNH: Wnt and hedgehog gene pathway expression in serous ovarian cancer. Int J Gynecol Cancer 21: 975-980, 2011.

19. Yang L, He J, Huang S, Zhang X, Bian Y, He N, Zhang H and Xie J: Activation of hedgehog signaling is not a frequent event in ovarian cancers. Mol Cancer 8: 112, 2009.

20. Ciucci A, De Stefano I, Vellone VG, Lisi L, Bottoni C, Scambia G, Zannoni GF and Gallo D: Expression of the glioma-associated oncogene homolog 1 (gli1) in advanced serous ovarian cancer is associated with unfavorable overall survival. PLoS One 8: e60145, 2013.

21. Chen Q, Xu R, Zeng C, Lu Q, Huang D, Shi C, Zhang W, Deng L, Yan R, Rao H, et al: Down-regulation of Gli transcription factor leads to the inhibition of migration and invasion of ovarian cancer cells via integrin $\beta 4$-mediated FAK signaling. PLoS One 9: e88386, 2014.

22. Steg AD, Burke MR, Amm HM, Katre AA, Dobbin ZC, Jeong DH and Landen CN: Proteasome inhibition reverses hedgehog inhibitor and taxane resistance in ovarian cancer. Oncotarget 5: 7065-7080, 2014. 
23. McCann CK, Growdon WB, Kulkarni-Datar K, Curley MD, Friel AM, Proctor JL, Sheikh H, Deyneko I, Ferguson JA, Vathipadiekal V, et al: Inhibition of Hedgehog signaling antagonizes serous ovarian cancer growth in a primary xenograft model. PLoS One 6: e28077, 2011.

24. Kandala PK and Srivastava SK: Diindolylmethane-mediated Gli1 protein suppression induces anoikis in ovarian cancer cells in vitro and blocks tumor formation ability in vivo. J Biol Chem 287: 28745-28754, 2012.

25. Ray A, Meng E, Reed E, Shevde LA and Rocconi RP: Hedgehog signaling pathway regulates the growth of ovarian cancer spheroid forming cells. Int J Oncol 39: 797-804, 2011.

26. Byrom J, Mudaliar V, Redman CW, Jones P, Strange RC and Hoban PR: Loss of heterozygosity at chromosome 9q22-31 is a frequent and early event in ovarian tumors. Int J Oncol 24 1271-1277, 2004

27. Tsuji T, Catasus L and Prat J: Is loss of heterozygosity at 9q22.3 (PTCH gene) and 19p13.3 (STK11 gene) involved in the pathogenesis of ovarian stromal tumors? Hum Pathol 36: 792-796, 2005.

28. Levanat S, Musani V, Komar A and Oreskovic S: Role of the hedgehog/patched signaling pathway in oncogenesis: A new polymorphism in the PTCH gene in ovarian fibroma. Ann NY Acad Sci 1030: 134-143, 2004

29. Levanat S, Kappler R, Hemmerlein B, Döring P, Musani V, Komar A, Oreskovic S, Pavelic B and Hahn H: Analysis of the PTCH1 signaling pathway in ovarian dermoids. Int J Mol Med 14: 793-799, 2004

30. Musani V, Sabol M, Car D, Ozretić P, Kalafatić D, Maurac I, Orešković S and Levanat S: PTCH1 gene polymorphisms in ovarian tumors: Potential protective role of c.3944T allele. Gene 517: 55-59, 2013

31. Cretnik M, Musani V, Oreskovic S, Leovic D and Levanat S: The Patched gene is epigenetically regulated in ovarian dermoids and fibromas, but not in basocellular carcinomas. Int J Mol Med 19: 875-883, 2007.

32. Löf-Öhlin ZM, Levanat S, Sabol M, Sorbe B and Nilsson TK: Promoter methylation in the PTCH gene in cervical epithelial cancer and ovarian cancer tissue as studied by eight novel Pyrosequencing ${ }^{\circledR}$ assays. Int J Oncol 38: 685-692, 2011.

33. Maurac I, Sabol M, Musani V, Car D, Ozretic P, Kalafatic D, Oreskovic S, Babic D and Levanat S: A low-grade ovarian carcinoma case with coincident LOH of PTCH1 and BRCA1, and a mutation in BRCA1. Int J Gynecol Pathol 31: 264-271, 2012.

34. Leovic D, Sabol M, Ozretic P, Musani V, Car D, Marjanovic K, Zubcic V, Sabol I, Sikora M, Grce M, et al: Hh-Gli signaling pathway activity in oral and oropharyngeal squamous cell carcinoma. Head Neck 34: 104-112, 2012.

35. McCall MN, McMurray HR, Land H and Almudevar A: On nondetects in qPCR data. Bioinformatics 30: 2310-2316, 2014.

36. Colton T: Statistics in Medicine. Little Brown \& Co., Boston, 1974.
37. Xing J, Jia C-R, Wang Y, Guo J and Cai Y: Effect of shRNA targeting survivin on ovarian cancer. J Cancer Res Clin Oncol 138: 1221-1229, 2012.

38. Kurman RJ and Shih IeM: The origin and pathogenesis of epithelial ovarian cancer: A proposed unifying theory. Am J Surg Pathol 34: 433-443, 2010.

39. Jope RS: Lithium and GSK-3: One inhibitor, two inhibitory actions, multiple outcomes. Trends Pharmacol Sci 24: 441-443, 2003.

40. Stecca B and Ruiz I Altaba A: Context-dependent regulation of the GLI code in cancer by HEDGEHOG and non-HEDGEHOG signals. J Mol Cell Biol 2: 84-95, 2010.

41. Koshiyama M, Matsumura N and Konishi I: Recent concepts of ovarian carcinogenesis: type I and type II. Biomed Res Int 2014: 934261, 2014

42. Sabol M, Trnski D, Uzarevic Z, Ozretic P, Musani V, Rafaj M, Cindric $M$ and Levanat S: Combination of cyclopamine and tamoxifen promotes survival and migration of mef-7 breast cancer cells - interaction of hedgehog-gli and estrogen receptor signaling pathways. PLoS One 9: e114510, 2014.

43. Shakoori A, Ougolkov A, Yu ZW, Zhang B, Modarressi MH, Billadeau DD, Mai M, Takahashi Y and Minamoto T: Deregulated GSK3 $\beta$ activity in colorectal cancer: Its association with tumor cell survival and proliferation. Biochem Biophys Res Commun 334: 1365-1373, 2005.

44. Trnski D, Sabol M, Gojević A, Martinić M, Ozretić P, Musani V, Ramić S and Levanat S: GSK3 $\beta$ and Gli3 play a role in activation of Hedgehog-Gli pathway in human colon cancer - Targeting GSK3 $\beta$ downregulates the signaling pathway and reduces cell proliferation. Biochim Biophys Acta 1852: 2574-2584, 2015.

45. Rask K, Nilsson A, Brännström M, Carlsson P, Hellberg P, Janson PO, Hedin L and Sundfeldt K: Wnt-signalling pathway in ovarian epithelial tumours: Increased expression of $\beta$-catenin and GSK33. Br J Cancer 89: 1298-1304, 2003.

46. Hilliard TS, Gaisina IN, Muehlbauer AG, Gaisin AM, Gallier F and Burdette JE: Glycogen synthase kinase $3 \beta$ inhibitors induce apoptosis in ovarian cancer cells and inhibit in-vivo tumor growth. Anticancer Drugs 22: 978-985, 2011.

47. Lauth M, Bergström A, Shimokawa T and Toftgård R: Inhibition of GLI-mediated transcription and tumor cell growth by smallmolecule antagonists. Proc Natl Acad Sci USA 104: 8455-8460, 2007.

48. Tostar U, Toftgård R, Zaphiropoulos PG and Shimokawa T: Reduction of human embryonal rhabdomyosarcoma tumor growth by inhibition of the hedgehog signaling pathway. Genes Cancer 1: 941-951, 2010

49. Gonnissen A, Isebaert S and Haustermans $\mathrm{K}$ : Targeting the Hedgehog signaling pathway in cancer: Beyond Smoothened. Oncotarget 6: 13899-13913, 2015.

50. Elias KM, Emori MM, Papp E, MacDuffie E, Konecny GE, Velculescu VE and Drapkin R: Beyond genomics: Critical evaluation of cell line utility for ovarian cancer research. Gynecol Oncol 139: 97-103, 2015. 